\title{
Poly(glutamic acid) nanofibre modified glassy carbon electrode: Characterization by atomic force microscopy, voltammetry and electrochemical impedance
}

\author{
Daniela Pereira Santos ${ }^{\mathrm{a}}$, Maria Valnice Boldrin Zanoni ${ }^{\mathrm{a}}$, Márcio Fernando Bergamini ${ }^{\mathrm{a}}$, \\ Ana-Maria Chiorcea-Paquim ${ }^{b}$, Victor Constantin Diculescu ${ }^{\mathrm{b}}$, Ana-Maria Oliveira Brett ${ }^{\mathrm{b}, *}$ \\ ${ }^{a}$ Departamento de Química Analítica, Instituto de Química, Universidade Estadual Paulista, Caixa Postal 355, 14800-900 Araraquara, S.P., Brazil \\ ${ }^{\mathrm{b}}$ Departamento de Química, Faculdade de Ciências e Tecnologia, Universidade de Coimbra, 3004-535 Coimbra, Portugal
}

Received 21 May 2007; received in revised form 2 August 2007; accepted 29 August 2007

Available online 6 September 2007

\begin{abstract}
Glassy carbon electrodes (GCE) were modified with poly(glutamic acid) acid films prepared using three different procedures: glutamic acid monomer electropolymerization (MONO), evaporation of poly(glutamic acid) (PAG) and evaporation of a mixture of poly(glutamic acid)/glutaraldehyde (PAG/GLU). All three films showed good adherence to the electrode surface. The performance of the modified GCE was investigated by cyclic voltammetry and differential pulse voltammetry, and the films were characterized by atomic force microscopy (AFM) and electrochemical impedance spectroscopy (EIS). The three poly(glutamic acid) modified GCEs were tested using the electrochemical oxidation of ascorbic acid and a decrease of the overpotential and the improvement of the oxidation peak current was observed. The PAG modified electrode surfaces gave the best results. AFM morphological images showed a polymeric network film formed by well-defined nanofibres that may undergo extensive swelling in solution, allowing an easier electron transfer and higher oxidation peaks.
\end{abstract}

(C) 2007 Elsevier Ltd. All rights reserved.

Keywords: Poly(glutamic acid) (PAG); Atomic force microscopy (AFM); Electrochemical impedance spectroscopy (EIS); Modified electrode; Nanofibres

\section{Introduction}

The successful use of synthetic polyaminoacid films on electrode surfaces to electrostatically attract and bind ionic molecules bearing opposite charges to that of the polyelectrolyte or complexing sites has received much attention. This interest is due to their importance as simplified models of natural polyelectrolytes such as proteins and nucleic acids, and the diversity of functional groups present in polyaminoacids with different analytical and clinical applications. Another advantage is the polyaminoacid film immobilisation procedure on the electrode surface that is extremely simple, rapid and can to be considered as an excellent alternative to manufacture electrochemical sensors.

In the literature there are many examples of electrochemical sensors applications based on the immobilization of polylysine

\footnotetext{
* Corresponding author.

E-mail address: brett@ci.uc.pt (A.-M. Oliveira Brett).
}

[1-10], polyalanine [11], polyglycine [12,13], poly(glutamic acid) [14-18], polyhystidine [19-22], polycysteine [23-25], polytyrosine [26] and polytryptofane [27,28]. Together with their ease of preparation, good stability, reproducibility, they show improved selectivity and sensitivity in electroanalytical measurements.

The synthetic polyaminoacid film modified electrode is also particularly advantageous to examine the kinetics of redox selfexchange reactions [2]. Some of the first reports in this area involved the preparation of electrodes with poly(L-lysine) films (PLL) [1-3], where protonated PLL deposited on a graphite electrode was employed to study the mechanism of charge transport within the film. Theoretical models explained how the PLL films present a porous structure and undergo very extensive swelling in aqueous electrolytes [2]. This feature accentuated the role of the reactants that are dissolved in the solution and can occupy the empty spaces within the swollen film. The model to explain the charge propagation within the polyelectrolyte films was based on the existence of two phases [2]. The first phase consisted of the region where the counterions were constrained by electrostatic 
(A)<smiles>NC(CCC(=O)O)C(=O)O</smiles><smiles>CCOC(=O)C(CCC(=O)O)NOC(=O)C(CCC(=O)O)NOC(=O)C(CCC(=O)O)NC(=O)O</smiles>

Scheme 1. Chemical structures: (A) glutamic acid and (B) poly(glutamic acid) (PAG).

forces to remain in the vicinity of the fixed-charge centres in the polyelectrolyte. The second phase comprised the remaining volume of the film that is assumed to be occupied by the supporting electrolyte solution containing both electroactive and electroinactive counterions. The coupling between these two propagation pathways by means of electron exchange is particularly favourable for rapid motion of counterions in the open, swollen structure of the film. Therefore, the PLL electrode proved to be very effective in facilitating electron transfer of several negatively charged analytes or to pre-concentrate them, by electrostatic interaction or complexation, proteins, transition metal complexes, pharmaceutical compounds, dyes and others [1-3].

Poly(glutamic acid) (PAG), see structure in Scheme 1, is a typical synthetic polyaminoacid rarely used to construct electrochemical sensors, although its structure can be very relevant as a biopolymer model. This polymer displays chains of biodegradable and controllable size of the naturally occurring L-glutamic acid linked together through amide bonds. Repetitive units linked between the $\alpha$-amino and $\delta$-carboxylic acid functional groups [29] and free protonated carboxylic groups $\left(\mathrm{p} K_{\mathrm{a}}=4.07\right)$ can be easily deposited on an electrode surface. The films of PAG generated by direct electrooxidation of their monomer are commonly employed in the determination of hydrazine, dopamine, ascorbic acid and caffeic acid [14-18]. Recently, we have described how PAG films can also be obtained by direct deposition of the polymer on the electrode surface followed by the evaporation of the solvent and cross-linked reaction with glutaraldehyde [18]. These previous results have indicated that the procedure adopted during film construction can interfere drastically in the subsequent voltammetric response of the analyte and its electrocatalytic response, but there is no study in the literature concerning the electrochemical characteristics of the film prepared by this method.

This paper presents the comparison between the performances of poly(glutamic acid) films modified glassy carbon electrode (GCE) surfaces prepared by three different procedures. The characterisation was done using atomic force microscopy (AFM), voltammetry and electrochemical impedance spectroscopy (EIS). These techniques have been successfully used to investigate the microscopic properties of the modified electrode surfaces and to obtain detailed information about the interface [29-33]. The oxidation of ascorbic acid (AA), an important bioactive substance, which reaction is electrocatalyzed at poly (glutamic acid) film modified GCEs [18] was used as model. A further advantage of the method proposed to modify the GCE was to improve the sensitivity and decrease the surface fouling in comparison with bare glassy carbon electrodes $[35,36]$.

\section{Experimental}

\subsection{Chemicals and solutions}

Suprapur grade reagents supplied by Merck and purified water from a Millipore Milli-Q system (conductivity $\leq 0.1 \mu \mathrm{S} \mathrm{cm}^{-1}$ ) were used in the preparation of all 
Table 1

Buffer solutions used as supporting electrolyte

\begin{tabular}{llr}
\hline Solution 1 & Solution 2 & pH \\
\hline $0.2 \mathrm{M} \mathrm{KCl}$ & $0.2 \mathrm{M} \mathrm{HCl}$ & 1.2 \\
$0.2 \mathrm{M} \mathrm{KCl}$ & $0.2 \mathrm{M} \mathrm{HCl}$ & 2.0 \\
$0.2 \mathrm{M} \mathrm{NaOAc}$ & $0.2 \mathrm{M} \mathrm{HAcO}$ & 3.4 \\
$0.2 \mathrm{M} \mathrm{NaOAc}$ & $0.2 \mathrm{M} \mathrm{HAcO}$ & 4.3 \\
$0.2 \mathrm{M} \mathrm{NaOAc}_{0.2 \mathrm{M} \mathrm{Na}_{2} \mathrm{HPO}_{4}}$ & $0.2 \mathrm{M} \mathrm{HAcO}^{2}$ & 5.0 \\
$0.2 \mathrm{M} \mathrm{Na}_{2} \mathrm{HPO}_{4}$ & $0.2 \mathrm{M} \mathrm{NaH}_{2} \mathrm{PO}_{4}$ & 6.0 \\
$0.2 \mathrm{M} \mathrm{Na}_{2} \mathrm{HPO}_{4}$ & $0.2 \mathrm{M} \mathrm{NaH}_{2} \mathrm{PO}_{4}$ & 6.9 \\
\hline
\end{tabular}

solutions. Buffer solutions used as supporting electrolytes in the experiments are shown in Table 1. Ascorbic acid (AA) solutions were prepared daily using dried pure analytica grade PA. Poly(glutamic acid) (PAG) $(\mathrm{MM}=50,000$, Aldrich) solution $1 \%(\mathrm{w} / \mathrm{v})$ and glutaraldehyde (GLU) solution $25 \%$ $(\mathrm{v} / \mathrm{v}$, Aldrich) in water were utilized in all experiments.

The $\mathrm{pH}$ measurements were carried out using a Micronal $\mathrm{pH}$ meter B222 model with a Micronal combined $\mathrm{pH}$ reference electrode.

All experiments were done at room temperature $\left(25 \pm 1^{\circ} \mathrm{C}\right)$.

\subsection{Preparation of poly(glutamic acid) modified carbon electrodes}

The poly(glutamic acid) films were prepared on carbon electrodes: glassy carbon (GCE) or highly oriented pyrolytic graphite (HOPG), using three different procedures: (1) glutamic acid monomer electropolymerization (MONO), (2) evaporation of poly(glutamic acid) (PAG) and (3) evaporation of poly(glutamic acid) and glutaraldehyde (PAG/GLU). A GCE was used in the voltammetric and impedance studies and the GCE or HOPG in the AFM experiments.

\subsubsection{Procedure 1 (MONO modified GCE)}

The electrode was modified using glutamic acid monomer electropolymerization. The clean GCE was immersed in a $0.02 \mathrm{M}$ glutamic acid solution in $\mathrm{pH} 7.00 .1 \mathrm{M}$ phosphate buffer $[13,14]$. Successively CVs were recorded between -0.8 and $+2.0 \mathrm{~V}\left(15\right.$ cycles, at $\left.100 \mathrm{mV} \mathrm{s}^{-1}\right)$. Afterwards, the electrode was gently washed with water and dried in a sterile atmosphere at room temperature.

\subsubsection{Procedure 2 (PAG modified GCE and $H O P G$ )}

For the voltammetric experiments, an aliquot of $16 \mu \mathrm{L}$ of an aqueous polymeric solution of PAG (1\%, w/v) was placed on the polished GCE surface. Both modified GCE and HOPG were used in AFM experiments. They presented a larger surface area, and $20 \mu \mathrm{L}$ poly(glutamic acid) $(1 \%$, w/v) was used to prepare the PAG modified electrodes, using the tip of a pipette to spread the poly(glutamic acid) over the surfaces. The PAG modified GCE and HOPG electrodes were then set aside and allowed to dry in a sterile atmosphere at room temperature.

\subsubsection{Procedure 3 (PAG/GLU modified GCE)}

The third method used to modify the electrode was based on the product of the reaction between poly(glutamic acid) (PAG) $(1 \%, \mathrm{w} / \mathrm{v})$ and glutaraldehyde (GLU) $(0.05 \%, \mathrm{v} / \mathrm{v})$ solution [10]. For voltammetric experiments, an aliquot of $4 \mu \mathrm{L}$ of the GLU solution was placed on the polished GCE surface. The tip of a pipette was used to spread the solution evenly over the whole surface to which $12 \mu \mathrm{L}$ of the PAG solution were added, followed by the addition of a further $4 \mu \mathrm{L}$ of GLU solution. The solutions were mixed with the tip of the pipette directly on the electrode surface. The in situ prepared PAG/GLU modified GCE was then set aside and allowed to dry in a sterile atmosphere at room temperature. For the AFM experiments, the same procedure was used, except that the volume of PAG solution was increased to $15 \mu \mathrm{L}$ and the volume of GLU to $5 \mu \mathrm{L}$.

The AFM images were taken immediately after the preparation of poly(glutamic acid) modified GCE and HOPG electrodes.

The voltammetric experiments with the PAG modified GCE and PAG/GLU modified GCE were submitted to a conditioning procedure in $\mathrm{pH} 7.00 .1 \mathrm{M}$ phosphate buffer and successive $\mathrm{CVs}$ were recorded between -0.8 and $+2.0 \mathrm{~V}$ (15 cycles, at $100 \mathrm{mV} \mathrm{s}^{-1}$ ). Then the electrodes were washed and transferred into solutions containing supporting electrolyte with or without AA, which was used as the model compound.

\subsection{Atomic force microscopy}

The GCE, approximately $6 \mathrm{~mm}$ diameter, and HOPG, grade $\mathrm{ZYB}$ of $15 \mathrm{~mm} \times 15 \mathrm{~mm} \times 2 \mathrm{~mm}$ dimensions, from Advanced Ceramics Co., were used as substrates in the AFM study.

The GCE was polished using diamond spray (particle size $1 \mu \mathrm{m}$ ) before every AFM assay. After polishing, the electrode was rinsed thoroughly with Milli-Q water for $30 \mathrm{~s}$. The HOPG was freshly cleaved with adhesive tape prior to each experiment and imaged by MAC Mode AFM in order to establish its cleanliness.

The MONO modified GCE was prepared in a onecompartment Teflon cell of approximately $12.5 \mathrm{~mm}$ internal diameter, holding the GCE at the bottom of the cell. A Pt wire counter electrode and an Ag wire as quasi-reference electrode (AgQRE) were placed in the cell, dipping approximately $5 \mathrm{~mm}$ into the solution. The electrochemical experiments were carried out using a $\mu$ Autolab running with GPES 4.9 software, EcoChemie, Utrecht, The Netherlands. A scan rate $v=100 \mathrm{mV} \mathrm{s}^{-1}$ was used.

AFM was performed with a PicoSPM equipment controlled by a MAC Mode module and interfaced with a PicoScan controller from Molecular Imaging Corp., Tempe, AZ. All the AFM experiments were performed with a CS AFM S scanner with a scan range $6 \mu \mathrm{m}$ in $x-y$ and $2 \mu \mathrm{m}$ in $z$, from Molecular Imaging Corporation. Silicon type II MAClevers of $225 \mu \mathrm{m}$ length, $2.8 \mathrm{~N} \mathrm{~m}^{-1}$ spring constants and $60-90 \mathrm{kHz}$ resonant frequencies in air and $27-30 \mathrm{kHz}$ resonant frequencies in liquid (Molecular Imaging Co.) were used. All images (256 samples/line $\times 256$ lines) were taken at room temperature; scan rates $0.8-1.6$ lines s $^{-1}$. When necessary, MAC Mode AFM images were processed by flattening in order to remove 
the background slope and the contrast and brightness were adjusted.

\subsection{Voltammetry and electrochemical impedance spectroscopy}

The voltammetric experiments were carried out using a $\mu$ Autolab running with GPES 4.9 software, Eco-Chemie, Utrecht, The Netherlands. The experimental conditions for differential pulse voltammetry (DPV) were: pulse amplitude $50 \mathrm{mV}$, pulse width $70 \mathrm{~ms}$, scan rate $5 \mathrm{mV} \mathrm{s}^{-1}$. Cyclic voltammograms (CVs) were recorded at scan rate $\nu=100 \mathrm{mV} \mathrm{s}^{-1}$, unless otherwise stated. The electrochemical impedance measurements were carried out using a FRA software version 4.9 , and a rootmean-square (r.m.s.) perturbation of $5 \mathrm{mV}$ was applied. The data were collected for 70 harmonic frequencies from 100 to $0.01 \mathrm{~Hz}$ at $10 \mathrm{steps} / \mathrm{dec}$ ade, with a polarization potential of $+0.2 \mathrm{~V}$ vs. SCE.

A system of three electrodes (EG\&G PARC) was also used, which consisted of an SCE as reference electrode, a platinum wire as auxiliary and a glassy carbon electrode (GCE, $3 \mathrm{~mm}$ diameter) as working electrode. The GCE was polished with alumina ( $0.3 \mathrm{~mm}$, BUEHLER).

All electrochemical measurements were performed in a Faraday cage.

\section{Results and discussion}

Atomic force microscopy (AFM), electrochemical impedance spectroscopy (EIS), cyclic voltammetry (CV) and differential pulse voltammetry (DPV) were used to understand the relationship between the morphological characteristics and the electrochemical properties of the GCE surface modified by poly(glutamic acid) using different preparation procedures: electropolymerization of the glutamic acid monomer (MONO), evaporation of the poly(glutamic acid) (PAG) and evaporation of an in situ prepared mixture of poly(glutamic acid) with glutaraldehyde (PAG/GLU), and the performance of the three poly(glutamic acid) modified electrodes prepared was tested using the electrochemical oxidation of ascorbic acid (AA) as model system.

\subsection{Atomic force microscopy}

The formation and morphological characterisation of the poly(glutamic acid) films obtained on the GCE following the three different procedures, MONO, PAG, and PAG/GLU, described in the experimental section, were investigated using AFM in air (Fig. 1). MAC Mode AFM was used throughout this study, because it is a gentle non-damaging technique that allows the visualization of the molecules and films
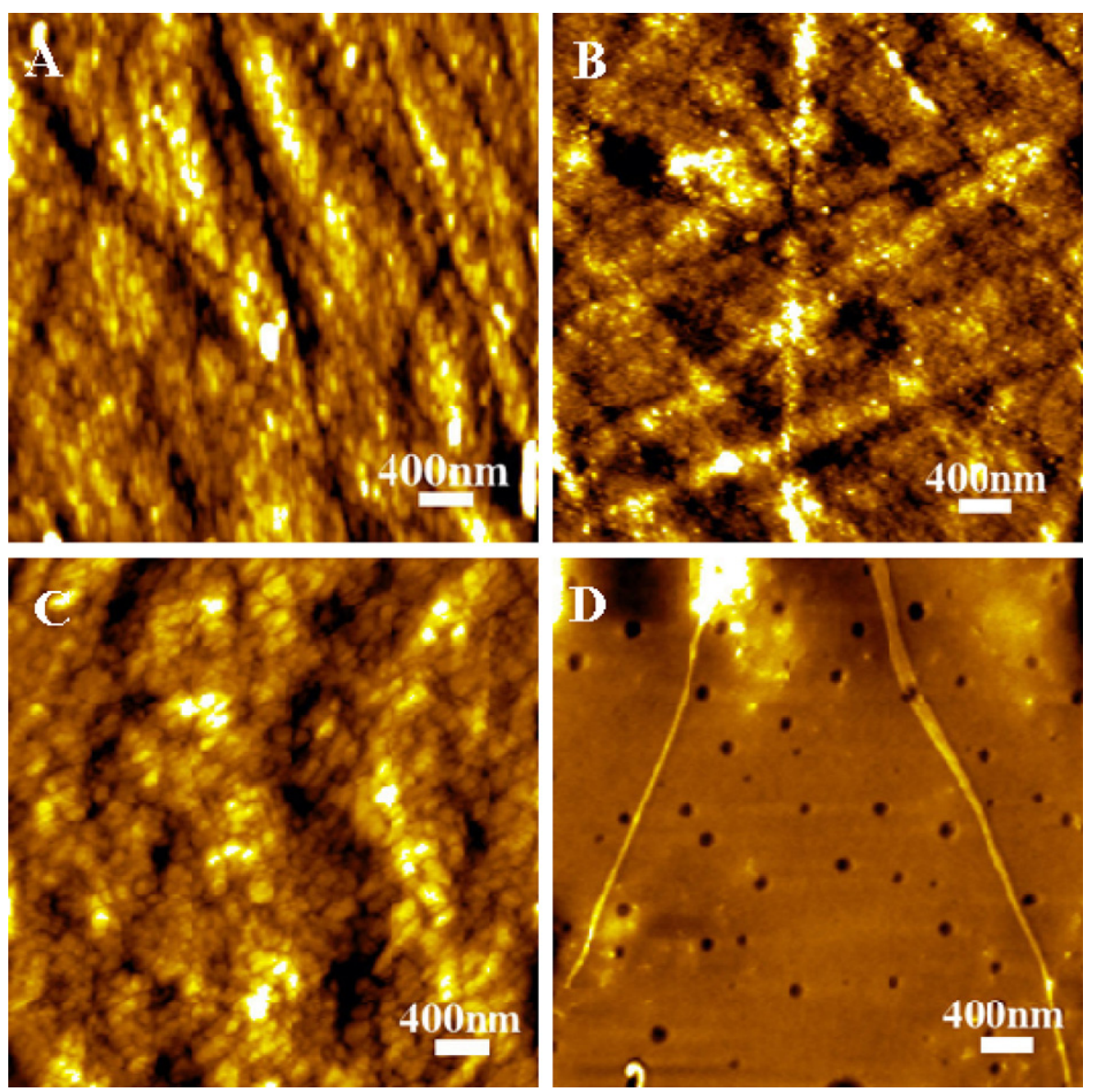

Fig. 1. MAC Mode AFM topographical images in air: (A) bare GCE, and after modification, (B) MONO modified GCE, (C) PAG modified GCE, (D) PAG/GLU modified GCE. 
that were attached on the electrode surface by soft interactions.

The AFM images of the GCE surface obtained before (Fig. 1A), and after modification (Fig. 1B-D), by the different poly(glutamic acid) film preparation methods will be evaluated.

The MONO modified GCE obtained by the electropolymerization of the glutamic acid monomer in $0.02 \mathrm{M}$ solution concentration allowed the formation of a thin PAG film on the surface of the electrode. Indeed, the AFM images demonstrated that the MONO modified GCE presented a thin granular topography (Fig. 1B) with the characteristic stripes of the bare GCE surface still visible.

Mono-glutamic acid has $\mathrm{p} K_{\mathrm{a}}=4.1$, meaning that at $\mathrm{pH} 6$, used in this study, it will be deprotonated $\left(\mathrm{COO}^{-}\right)$. The electrochemical oxidation of glutamic acid at $\mathrm{pH} 6$ occurs at $E_{\mathrm{p}}=+1.35 \mathrm{~V}$, the oxidation of the amino group to its corresponding radical cation. The oxidized amino group radical cation interacts with the glutamic acid monomer COO- group and polymerization occurs, Scheme 1. The PAG films generated by electropolymerization of the monomer are immobilized on the electrode surface [18].

However, after successive $\mathrm{CV}$ s recorded during film formation between -0.8 and $+2.0 \mathrm{~V}$, a decrease of the peak current at $+1.35 \mathrm{~V}$ was observed, meaning that few polyaminoacid chains were formed by the cross-linked reaction between the monomeric forms. Effectively, both AFM (Fig. 1B) and the voltammetric data gave evidence that the electrooxidation process led to a thin film, mixture of immobilized monomer and polymer glutamic acid.

On the contrary to what was observed for the MONO modified GCE, the formation of a thick, visible to the eye, multilayer film prepared by the evaporation of the corresponding solutions, both for the PAG and PAG/GLU modified GCEs (Fig. 1C and D) was obtained.

The PAG modified GCE films were thick and wrinkled (Fig. 1C) with uniform morphology and only very small pores that permitted the encapsulation of the analyte molecules into the film.

The PAG/GLU modified GCE films presented a very uniform and smooth morphology, with very well defined large pores, from 80 up to $180 \mathrm{~nm}$ width and $70-150 \mathrm{~nm}$ deep (Fig. 1D).
Although much smoother films with increased adhesion to the GCE electrode was achieved by PAG/GLU films, the presence of large pores on the modified surface can cause leakage of the analyte. These pores are due to the interactions between the poly(glutamic acid)-glutaraldehyde mixture with the carbon surface.

As observed in the AFM images (Fig. 1A), the GCE presents a rough topography, and insignificant differences were observed between the GCE and PAG-modified GCE electrodes. The PAG films followed the peaks and valleys present on the GCE surface, which makes observation of small morphological details difficult. For this reason, the morphological structure of PAG film was also studied on the HOPG surface (Fig. 2). The HOPG electrode is extremely smooth, inert in air and has easy to clean terraces on its basal plane, and allow a correct evaluation of the morphological characteristics of the immobilized films. Comparatively, for a $1000 \mathrm{~nm} \times 1000 \mathrm{~nm}$ surface area, the GCE has a r.m.s. roughness of $2.10 \mathrm{~nm}$, while the $\mathrm{HOPG}$ electrode surface has a r.m.s. roughness of less than $0.06 \mathrm{~nm}$ [37].

The high-resolution AFM images obtained, clearly showed a thick PAG multilayer film on the HOPG surface (Fig. 2A), formed by a polymeric network of nanofibres (Fig. 2B). This densely packed structure of PAG nanofibres causes a more effective incorporation of the analyte and the counterions into the film, a very useful characteristic for practical applications of the PAG modified GCE.

Besides AFM, the function-structure relationship of the poly (glutamic acid) modified GCE, using the three immobilization procedures previously described, was evaluated by cyclic voltammetry and impedance studies.

\subsection{Electrochemical oxidation of ascorbic acid on the modified GCE}

The MONO, PAG and PAG/GLU modified GCE were initially characterized by CV and DPV. After modification, each poly(glutamic acid) modified GCE was washed with deionized water, then transferred to $\mathrm{pH}$ 6.0 0.1 M acetate buffer and CVs recorded in the potential range from -0.2 to $+1.2 \mathrm{~V}$ showing no redox peaks (not shown). Thus these three poly(glutamic acid)
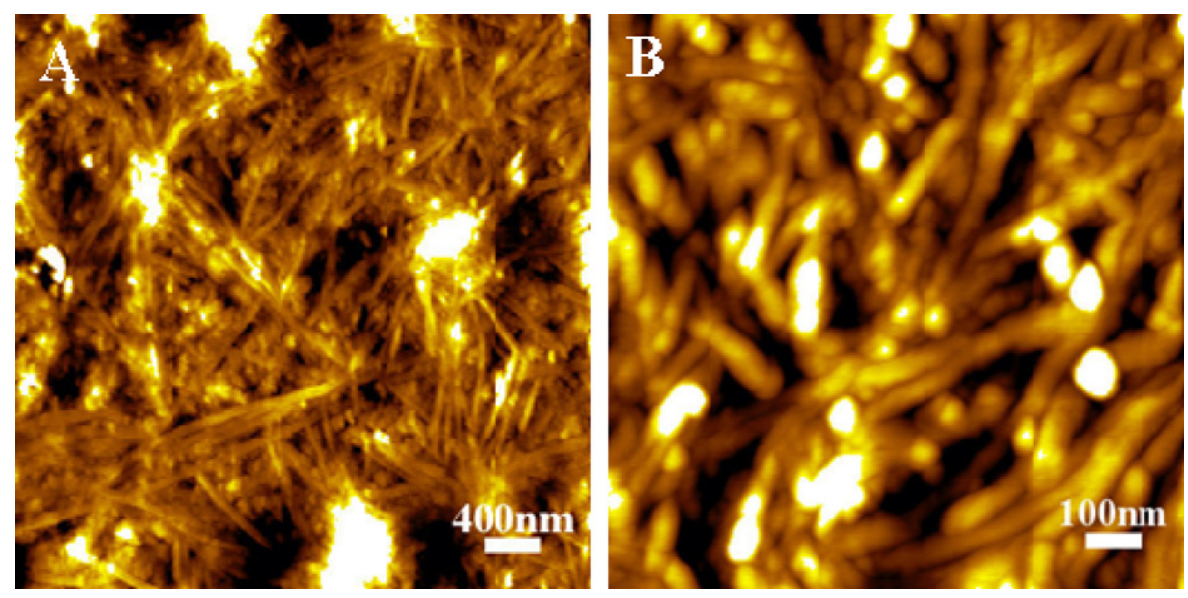

Fig. 2. MAC Mode AFM topographical images in air of the PAG modified HOPG surface. 


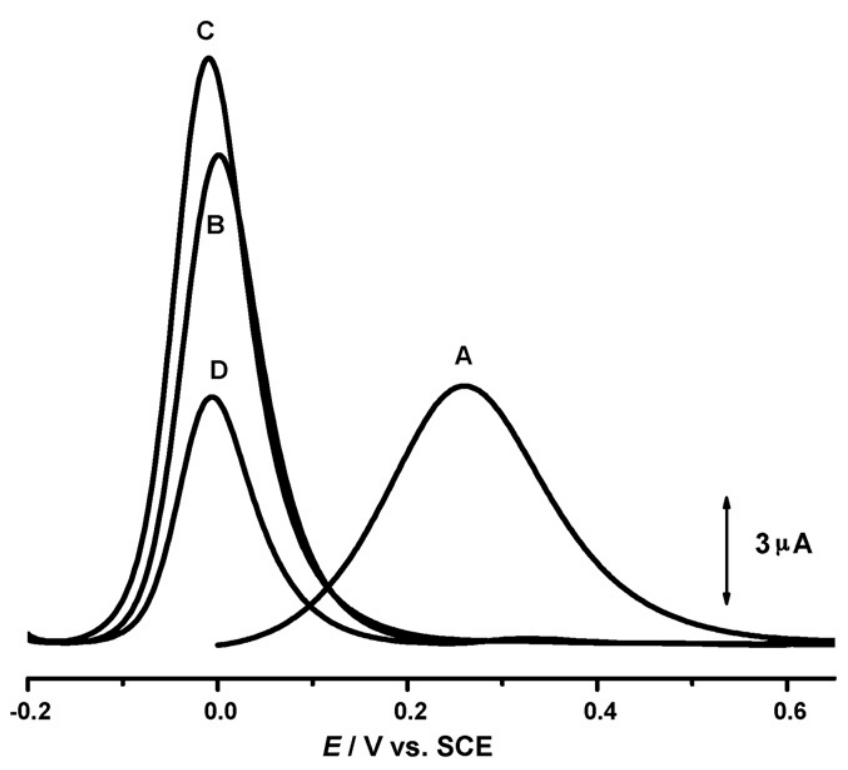

Fig. 3. DPVs obtained for $1 \mathrm{mM}$ AA in $\mathrm{pH} 7.00 .1 \mathrm{M}$ phosphate buffer: (A) bare GCE and after modification, (B) MONO modified GCE, (C) PAG modified GCE and (D) PAG/GLU modified GCE. Scan rate $=5 \mathrm{mV} \mathrm{s}^{-1}$.

modified GCEs provide a large potential window. Their performance was tested investigating the voltammetric oxidation behaviour of AA as model compound.

The electrochemical oxidation of $1 \mathrm{mM}$ AA in $\mathrm{pH} 6.00 .1 \mathrm{M}$ phosphate buffer was investigated by differential pulse voltammetry (DPV) using the bare GCE and the poly(glutamic acid) modified GCE (Fig. 3), obtained by the three procedures, MONO, PAG and PAG/GLU. The DVP of AA at bare GCE presented an oxidation peak at $E_{\mathrm{pa}}=+0.26 \mathrm{~V}$ which was shifted to a much less positive potential, $E_{\mathrm{pa}} \approx+0.00 \mathrm{~V}$, using any of the poly(glutamic acid) modified GCE. These results clearly show that the poly(glutamic acid) films caused a large decrease of the oxidation potential of AA.

The peak width at half height, $W_{1 / 2}$, and peak current on the DPVs in Fig. 3, changed. The value of $W_{1 / 2}=188 \mathrm{mV}$ obtained for DPVs recorded on the bare GCE, diminished on poly (glutamic acid) modified GCE to the values: $W_{1 / 2}=92 \mathrm{mV}$ for MONO modified GCE, $W_{1 / 2}=87 \mathrm{mV}$ for PAG modified GCE, and $W_{1 / 2}=96 \mathrm{mV}$ in the case of PAG/GLU modified GCE. Although high, the values obtained using the poly (glutamic acid) modified GCE are closer to the value expected for a reversible two-electron oxidation process of AA, than the value obtained with the bare GCE.

Although the decrease of the AA oxidation potential at PAG/GLU modified GCE is easily observed, the AA oxidation peak maintain the same current value observed when the DPV was recorded with the bare GCE. Nevertheless, an increase of the peak current was obtained when MONO modified GCE and PAG modified GCE were used. Therefore, it seems that the procedure adopted to cover the electrode surface is responsible for a variable and not reproducible film thickness which will cause the alterations observed on the peak currents recorded using CV and DPV, also in agreement with the films different morphology already investigated by AFM (Figs. 1 and 2).
The AFM experiments showed that the PAG modified GCE, gave rise to films with relatively large separations between the nanofibre polymer chains (Figs. 1C and 2), which can undergo very extensive swelling in solution. This polymeric network of well-defined polymeric chains separated as nanofibres was imaged by AFM (Fig. 2). The magnitude of the AA oxidation current at poly(glutamic acid) modified GCE was proportional to the rate at which ions can be carried across the film and the charge is easily propagated through the solution phase inside the porous film. Even for thinner films such as those obtained for the MONO modified GCE, the diffusion was also facilitated within the polymeric network.

A very thick film with well-defined large pores was formed on the PAG/GLU modified GCE surface, as found by AFM (Fig. 1D), which may cause leakage of AA from the pores. Therefore, the charge propagation through the PAG/GLU polyelectrolyte film may occur by a place-exchange reaction, the rate of which determines the resulting current.

This voltammetric behaviour of AA was also confirmed by $\mathrm{CV}$ where the peak potential, $E_{\mathrm{pa}}=+0.35 \mathrm{~V}$, using the bare GCE was shifted to $E_{\mathrm{pa}}=+0.08 \mathrm{~V}$ (not shown) at all poly(glutamic acid) modified GCEs. These results are similar to those verified for polyhistidine films described in the literature [22], where a decrease of $0.4 \mathrm{~V}$ in the oxidation potential of AA was observed. The analysis of the CV shape also showed the change of reversibility of the global process on poly (glutamic acid) modified electrodes. The value of $\alpha n_{\mathrm{a}}=0.43$ from $E_{\mathrm{pa}}-E_{\mathrm{p} / 2}=47.7 \mathrm{mV} / \alpha n_{\mathrm{a}}$, obtained from the $\mathrm{CV}$ at $\nu=50 \mathrm{mV} \mathrm{s}^{-1}$ with the bare GCE, changes to the values of 1.08 at MONO and PAG modified GCE, and 0.87 at PAG/GLU modified GCE.

The films prepared by the MONO, PAG and PAG/GLU procedures covered the GCE with a film that enables the retention of AA by entrapment near the surface of the electrode. To verify if the retention of AA by entrapment in the poly(glutamic acid) films was irreversible and caused a pre-concentration of AA, each different modified GCE was immersed in a continuously stirred solution of $1 \mathrm{mM} \mathrm{AA}$ in $\mathrm{pH}$ 6.0 $0.1 \mathrm{M}$ phosphate buffer for a fixed time. The CVs recorded after this procedure showed no change in the peak current for AA oxidation. This means that pre-concentration of AA by retention on the poly(glutamic acid) modified GCE surface did not occur.

Next, each MONO, PAG and PAG/GLU modified GCE prepared above was washed and placed in the electrochemical cell containing only pH 6.0 $0.1 \mathrm{M}$ phosphate buffer. The CVs recorded in these conditions showed no peak suggesting that no AA was retained in the inner layers of the poly(glutamic acid) films. This was followed by re-immersing each MONO, PAG and PAG/GLU modified GCE in the $1 \mathrm{mM}$ AA solution in $\mathrm{pH}$ 6.0 $0.1 \mathrm{M}$ phosphate buffer and on the CVs recorded the AA oxidation peak currents were restored.

The decrease of the overpotential for the oxidation of AA on the MONO, PAG and PAG/GLU modified GCE can be explained taking into consideration the diffusion of AA from the solution into the interior of the swollen MONO, PAG or PAG/GLU film that contains both electroactive and electroinactive counterions. The charge transfer is then facilitated due to propagation through 
the solution within the modified film layer [2]. The change in potential and anodic current observed for AA oxidation in these films could be attributed to a different accessibility of the AA within the film on the electrode surface. The more open the structure of the MONO, PAG and PAG/GLU films, the higher is the possibility to enable reactant diffusion within the inner domains to reach the electrode surface.

In order to obtain more information about the AA redox mechanism on a poly(glutamic acid) modified GCE, the electrochemical oxidation of AA was studied over a $\mathrm{pH}$ range between 1 and 8 using DPV. The DPVs (Fig. 4A) were all recorded in $1 \mathrm{mM}$ AA in different $\mathrm{pH}$ electrolyte solutions, $0.1 \mathrm{M}$ ionic strength. The AA oxidation peak potential was shifted to more negative values with increasing $\mathrm{pH}$ (Fig. 4B), and the slope of the dashed line, $59 \mathrm{mV}$ per $\mathrm{pH}$ unit, shows that the oxidation mechanism of AA involves the same number of electrons and protons.

The variation of peak current with $\mathrm{pH}$ (Fig. 4B) presents two maxima, for $\mathrm{pH} 2.0$ and 6.0. The electrostatic interaction with the polyelectrolyte that is in the protonated form will probably occur at $\mathrm{pH}$ 2.0. The increase of peak current obtained for $\mathrm{pH}$

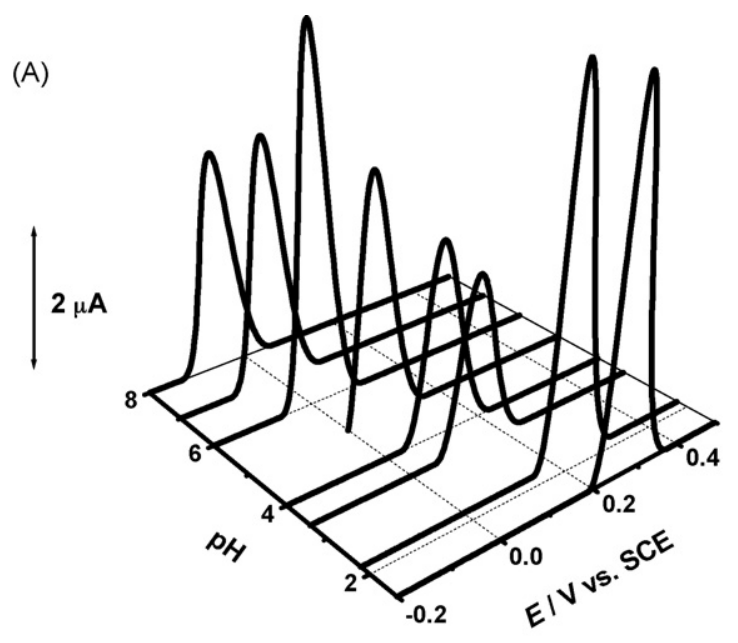

(B)

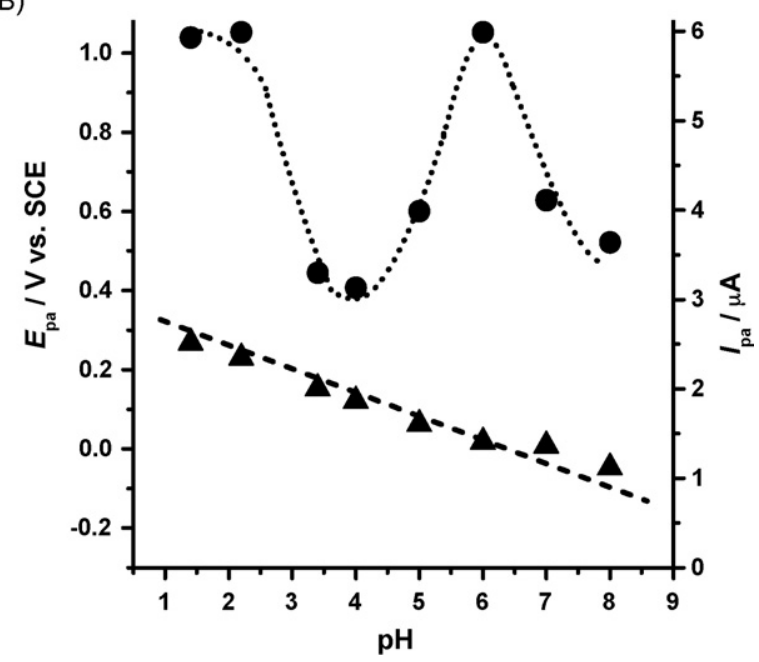

Fig. 4. (A) 3D plot of first DP voltammograms obtained in $1 \mathrm{mM}$ AA using a PAG modified GCE as a function of $\mathrm{pH}$. (B) Plot of $(\boldsymbol{\Lambda}) E_{\mathrm{pa}}$ and $(\bullet) I_{\mathrm{pa}} \mathrm{vs.} \mathrm{pH}$. Dashed line corresponds to a slope of $59 \mathrm{mV}$ per unit of $\mathrm{pH}$. Scan rate $=5 \mathrm{mV} \mathrm{s}^{-1}$.
6.0 suggests that other processes are aiding the electron transfer that cannot be attributed to electrostatic interaction, since both analyte and surface coating are in a non-protonated form. The same behaviour was obtained for MONO and PAG/GLU modified GCE in $1 \mathrm{mM}$ AA in $\mathrm{pH} 6.0$, although the oxidation peak currents were lower. This behaviour is evidence that the diffusion mechanism is optimized for the experimental conditions described, contributing to the transport of the analyte to the electrode surface around $\mathrm{pH} 6.0$ with improvement in the rate of electron transfer and, due to the higher peak current, the $\mathrm{pH} 6.0$ was chosen in subsequent AA electrochemical oxidation studies.

Considering that the contribution of the processes occurring inside and outside the polyelectrolyte domains could vary across the film, the influence of scan rate on the oxidation peak current of AA at MONO, PAG or PAG/GLU modified GCE was investigated in a $1 \mathrm{mM}$ AA solution in $\mathrm{pH}$ 6.0 $0.1 \mathrm{M}$ phosphate buffer. The values obtained were compared with those at a bare GCE. The plots of $I_{\mathrm{pa}}$ values vs. square root of the scan rate $\left(v^{1 / 2}\right)$ (Fig. 5), showed straight lines. This confirmed that the electrode process is controlled by diffusion through the polyelectrolyte film, which is sufficiently porous to allow AA to move through it and reach the GCE surface, in agreement with the AFM images (Figs. 1 and 2), but the film thickness can influence the diffusion rate. Also, Fig. 5 showed a different increase of the AA oxidation peak current with the scan rate depending on the modification procedure.

\subsection{Electrochemical impedance spectroscopy of ascorbic acid on the modified GCE}

Electrochemical impedance spectroscopy (EIS) is a simple and effective way to measure the charge transfer resistance $\left(R_{\mathrm{ct}}\right)$

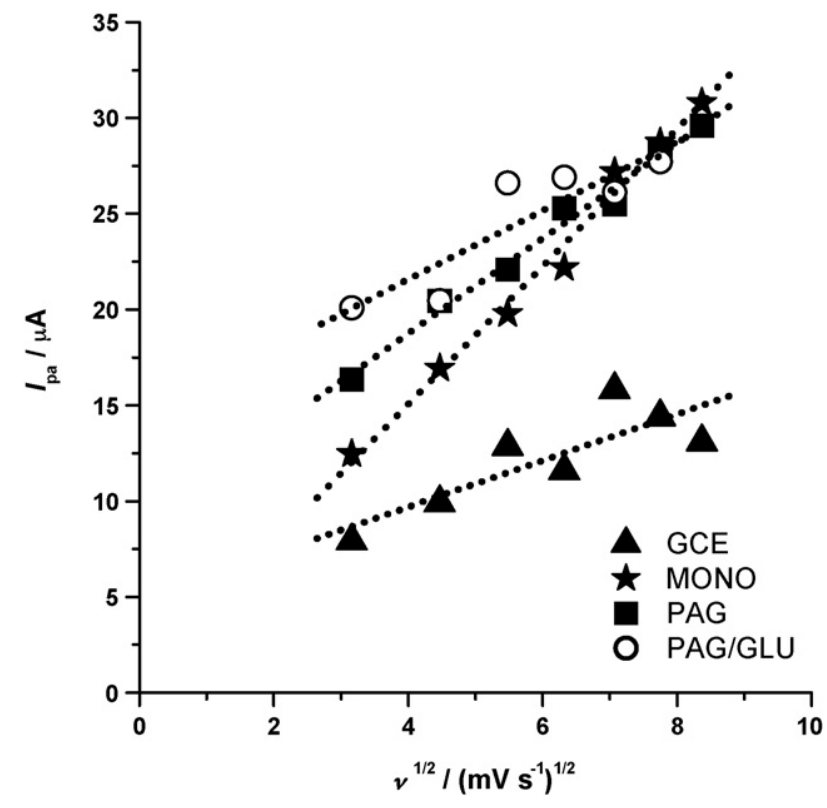

Fig. 5. Plot of peak current vs. square root of scan rate, obtained for $1 \mathrm{mM}$ AA in pH $6.00 .2 \mathrm{M}$ phosphate buffer: $(\boldsymbol{\Delta})$ bare GCE and after modification: $(\star)$ MONO modified GCE, (ם) PAG modified GCE and $(\bigcirc)$ PAG/GLU modified GCE. 


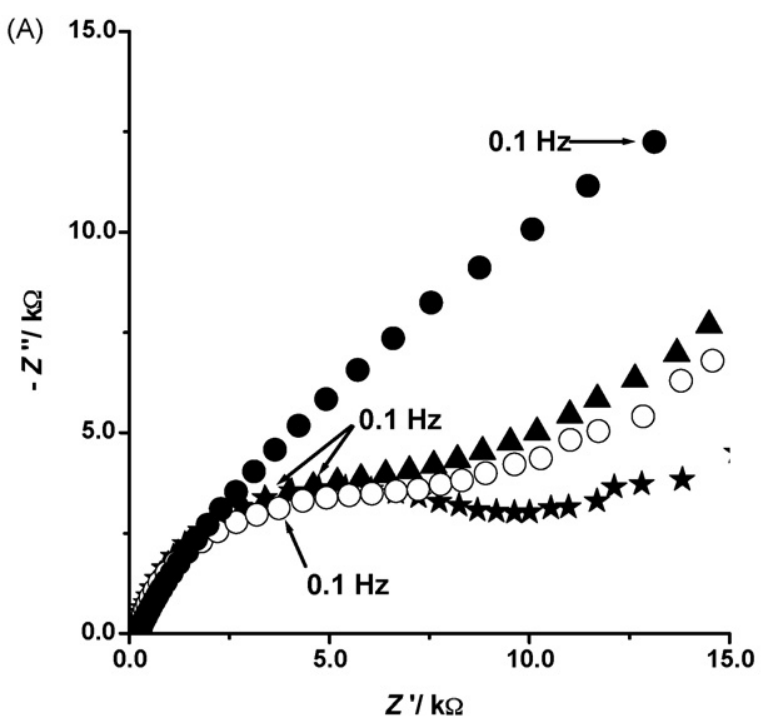

(B)

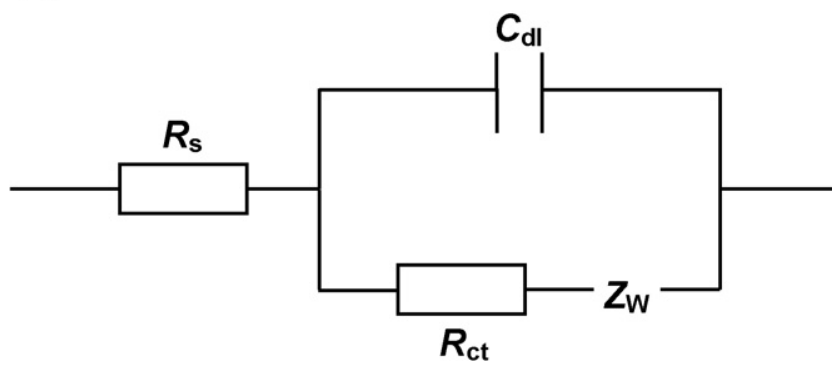

Fig. 6. (A) Complex plane plots of impedance spectra, $E=+0.02 \mathrm{~V}$, obtained for $1 \mathrm{mM}$ AA in $\mathrm{pH}$ 6.0 0.1 M phosphate buffer: $(-)$ bare GCE, and after modification: ( $\star$ ) MONO modified GCE, (ム) PAG modified GCE, and (O) PAG/GLU modified GCE. (B) Equivalent electrical circuit used to fit the impedance data obtained for MONO, PAG and PAG/GLU modified GCE.

of the electrochemical reactions [34]. The EIS measurements were performed in $1 \mathrm{mM} \mathrm{AA} \mathrm{pH} 6.00 .1 \mathrm{M}$ phosphate buffer solution using the bare GCE and poly(glutamic acid) modified GCE (Fig. 6A). It can be seen that each MONO, PAG and PAG/GLU modified GCE exhibit a semicircular and a linear portion.

The first semicircle corresponds to the charge transfer process through the film at high frequency range, whereas the second one is due to the diffusion process in the low frequency range. The diameter of the semicircle represents the magnitude of $R_{\mathrm{ct}}$ at the electrode surface. The measured EIS data were fitted with an equivalent circuit as shown in Fig. 6B. This equivalent circuit consists of the ohmic resistance $\left(R_{\mathrm{S}}\right)$ of the electrolyte solution, the double layer capacitance $\left(C_{\mathrm{dl}}\right)$, electron-transfer resistance $\left(R_{\mathrm{ct}}\right)$ and the Warburg impedance $\left(Z_{\mathrm{w}}\right)$ resulting from the diffusion of ions from the bulk of the electrolyte to the interface. This equivalent circuit was used to fit the impedance spectra and extract the values of $C_{\mathrm{dl}}$ and $R_{\mathrm{ct}}$.

In Table 2 are reported the fitted values of $R_{\mathrm{ct}}$ and $C_{\mathrm{dl}}$ for the bare GCE, MONO, PAG and PAG/GLU modified GCE. The $R_{\mathrm{ct}}$ for the bare GCE is $50.1 \mathrm{k} \Omega$, which is much higher than the $R_{\mathrm{ct}}$ values observed for MONO, PAG and PAG/GLU modified GCE. This low $R_{\mathrm{ct}}$ value for the modified electrodes implies
Table 2

Results for $R_{\mathrm{ct}}$ and $C_{\mathrm{dl}}$ obtained after fitting the electrochemical impedance spectra with the equivalent circuit

\begin{tabular}{lll}
\hline Electrode & $R_{\mathrm{ct}}(\mathrm{k} \Omega)$ & $C_{\mathrm{dl}}(\mu \mathrm{F})$ \\
\hline Bare GCE & 50.1 & 0.03 \\
MONO modified GCE & 13.96 & 3.8 \\
PAG modified GCE & 7.93 & 4.8 \\
PAG/GLU modified GCE & 7.59 & 3.6
\end{tabular}

that the charge transfer process is relatively fast compared to the bare GCE. It can also be observed from Table 2 that $C_{\mathrm{dl}}$ increases markedly when poly(glutamic acid) modified GCEs were used. Comparing the data obtained, it can be concluded that the PAG modified GCE presented the lowest $R_{\mathrm{ct}}$ in the interface and concomitant higher $C_{\mathrm{dl}}$, reflecting an improvement of the effective electron transfer rate.

\subsection{Electroanalysis of ascorbic acid on the modified GCE}

The analytical properties of the MONO, PAG and PAG/GLU modified GCE were evaluated for AA in $\mathrm{pH} 6.00 .1 \mathrm{M}$ phosphate buffer. Without accumulation time, calibration plots for the modified electrode were investigated from $10 \mu \mathrm{M}$ to $2.5 \mathrm{mM}$ using DPV (Fig. 7). The enhanced current at low concentrations of AA observed with each poly(glutamic acid) modified GCE, compared with that at the bare GCE, is quite clear, as was expected. The detection limit for AA using any of the poly(glutamic acid) modified electrodes was $1 \mu \mathrm{M}$. This concentration is two orders of magnitude lower than that at the bare GCE for the same experimental conditions.

The dependence on accumulation time of AA oxidation response using the three modification procedures was investigated also by $\mathrm{CV}$ for AA concentrations varying from $50 \mu \mathrm{M}$ to $2.5 \mathrm{mM}$. Each poly(glutamic acid) modified electrode was immersed in a cell containing AA in pH $6.00 .1 \mathrm{M}$ phosphate buffer for a constant time. Linear calibration graphs were

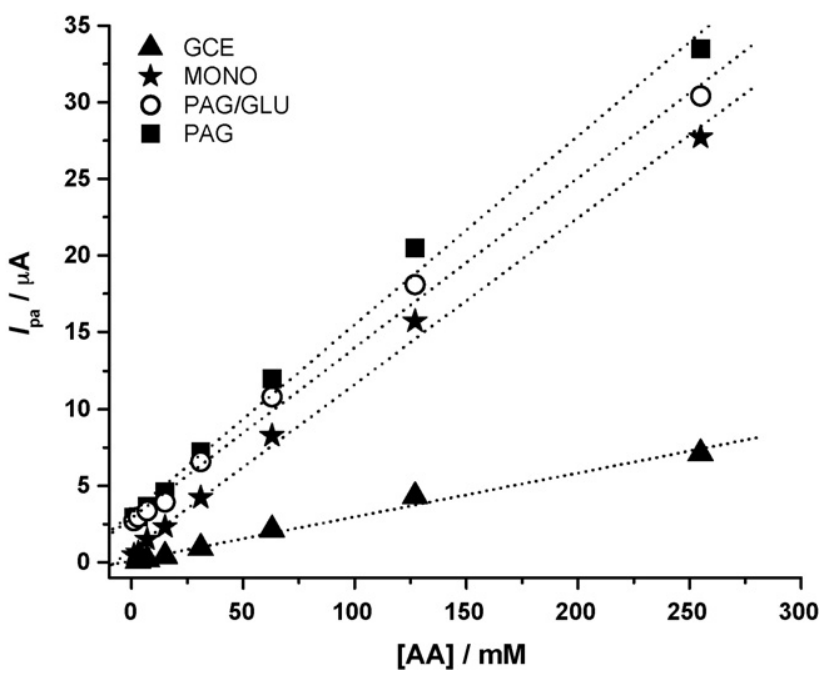

Fig. 7. Calibration plot for the determination of AA without accumulation time using DPV: $(\boldsymbol{\Delta})$ bare GCE and after modification: $(\star)$ MONO modified GCE, (a) PAG modified GCE and $(\bigcirc)$ PAG/GLU modified GCE. 
Table 3

Analytical parameters for AA concentrations varying from $50 \mu \mathrm{M}$ to $2.5 \mathrm{mM}$, in pH $6.00 .1 \mathrm{M}$ phosphate buffer, obtained from CV calibration plots using bare GCE, MONO, PAG and PAG/GLU modified GCEs

\begin{tabular}{lllll}
\hline Electrode & Equation $I_{\mathrm{pa}}(\mu \mathrm{A}), C\left(10^{-5} \mathrm{M}\right)$ & Linearity range $\left(10^{-5} \mathrm{M}\right)$ & Correlation coefficient & Detection limit $(\mu \mathrm{M})$ \\
\hline Bare GCE & $I_{\mathrm{pa}}=0.705+0.103 C$ & $15-127$ & 0.999 & 51.9 \\
MONO modified GCE & $I_{\mathrm{pa}}=2.174+0.195 C$ & $7-127$ & 0.999 & 19.9 \\
PAG modified GCE & $I_{\mathrm{pa}}=0.356+0.333 C$ & $3-63$ & 0.999 & 17.8 \\
PAG/GLU modified GCE & $I_{\mathrm{pa}}=2.338+0.254 C$ & $15-127$ & 0.996 & 13.9 \\
\hline
\end{tabular}

obtained on bare GCE, MONO, PAG and PAG/GLU modified GCE for AA by cyclic voltammetry (Table 3).

A slightly larger current was obtained for PAG modified GCE, the results indicating that probably the films generated by the PAG modification procedure improved the charge mechanism.

The important consequences of using a poly(glutamic acid) modified GCE are the low AA oxidation potential and lower detection limit compared to bare GCE, in agreement with the model of charge transportation in polyelectrolyte films proposed by Anson [2].

For thick films, such as for PAG/GLU, the reactant within the "Donnan domains" is predominantly pre-concentrated around the polyelectrolyte chain and carries most of the current. At pH 6.0 the electrostatic interaction is minimized and the peak current is lower than that obtained for thinner films. For thin films, such as MONO, the diffusion of the reactant confined inside the coating is favoured, since the access is easier, and less time is required for exchanging electrons or sites in the "Donnan domains". For films obtained using PAG deposition, the AFM studies confirmed that the film is formed by a network of nanofibre polymer chains (Fig. 2) in a way comparable to the model proposed by Anson [2]. The resulting current obtained for AA oxidation is the contribution from both processes, induced by the confined substrate inside and outside of the "Donnan domain" and favoured by rapid place-exchange reactions and the rapid motion of counterions in the open and swollen structure of the polyaminoacid coating.

\section{Conclusions}

In this study, three different procedures for the modification of GCE with poly(glutamic acid) (PAG) were characterised by atomic force microscopy, voltammetry and electrochemical impedance spectroscopy. The modifications of the GCE by monomeric glutamic acid electropolymerization (MONO), evaporation of poly(glutamic acid) (PAG) and evaporation of a mixture of poly(glutamic acid)/glutaraldehyde (PAG/GLU) lead to the formation of films with different morphologies on the electrode surface. The results clearly show that the poly(glutamic acid) modified electrodes present a polymeric network on its surface, allowing the decrease of the AA oxidation potential without adsorption of AA. The poly(glutamic acid) modified GCE led to a lower detection limit for AA than the bare GCE, this methodology thus showing good possibilities to construct a stable electrochemical sensor. The PAG modified GCE, with a morphology of very well-defined polymeric chains visualised as nanofibres, allowed an easier electron transfer and higher oxidation peaks, and presented the best results.

\section{Acknowledgments}

Financial support from FAPESP (Proc.: 03/06598-3), Fundação para a Ciência e Tecnologia (FCT), Post-Doctoral Grants SFRH/BPD/27087/2006 (A.-M. Chiorcea-Paquim), and SFRH/BPD/18824/2004 (V.C. Diculescu), POCI 2010 (cofinanced by the European Community Fund FEDER), and ICEMS (Research Unit 103), is gratefully acknowledged.

\section{References}

[1] F.C. Anson, T. Osaka, J.M. Saveant, J. Phys. Chem. 87 (1983) 640.

[2] F.C. Anson, J. Saveant, K. Shigwhara, J. Am. Chem Soc. 105 (1983) 1096.

[3] P. Bianco, J. Haladjian, S. Giannandrea-Derocles, Electroanalysis 6 (1994) 67.

[4] R.C.S. Luz, F.S. Damos, A.B. Oliveira, J. Beck, L.T. Kubota, Electrochim. Acta 50 (2005) 2675.

[5] R.C.S. Luz, F.S. Damos, A.B. Oliveira, J. Beck, L.T. Kubota, Sens. Actuators B 117 (2006) 274

[6] F.C. Pereira, A.G. Fogg, P. Ugo, E.P. Bergamo, N.R. Stradiotto, M.V.B. Zanoni, Electroanalysis 17 (2005) 1309.

[7] V.S. Fereira, M.V.B. Zanoni, A.G. Fogg, Anal. Chim. Acta 384 (1999) 159.

[8] E.R.C. Viana, F.C. Pereira, M.V.B. Zanoni, Dyes Pigm. 71 (2006) 145.

[9] S.C.C. Monterroso, H.M. Carapuça, A.C. Duarte, Talanta 68 (2006) 1655.

[10] F.C. Pereira, E.P. Bergamo, N.R. Stradiotto, M.V.B. Zanoni, A.G. Fogg, Electroanalysis 16 (2004) 1439.

[11] L. Zhang, Y.M. Sun, X. Lin, Analyst 126 (2001) 1760

[12] A.M. Yu, H.L. Zhang, H.Y. Chen, Electroanalysis 9 (1997) 788.

[13] L. Zhang, X. Lin, Fresenius, J. Anal. Chem. 370 (2001) 956.

[14] A.M. Yu, H.Y. Chen, Anal. Chim. Acta 344 (1997) 181.

[15] A.M. Yu, H.Y. Chen, Anal. Lett. 30 (1997) 599.

[16] M. Niwa, M. Matsui, K. Koide, N. Higashi, J. Mater. Chem. 7 (1997) 2191.

[17] L. Zhang, X. Lin, Analyst 126 (2001) 367.

[18] D.P. Santos, M.F. Bergamini, A.G. Fogg, M.V.B. Zanoni, Microchim. Acta 151 (2005) 127.

[19] Y.T. Long, H.Y. Chen, J. Electroanal. Chem. 440 (1997) 239.

[20] G. Li, L. Chen, J. Zhu, D. Zhu, D.F. Untereker, Electroanalysis 11 (1999) 139.

[21] J.C. Moreira, R. Zhao, A.G. Fogg, Analyst 115 (1990) 1561.

[22] A.M. Yu, D.M. Sun, H.Y. Gu, H.Y. Chen, Anal. Lett. 29 (1996) 2633.

[23] Y. Bai, X. Ruan, J. Mo, Y. Xie, Anal. Chim. Acta 373 (1998) 39.

[24] A.C. Liu, D.C. Chen, C.C. Lin, H.H. Chou, C.H. Chen, Anal. Chem. 71 (1999) 1549.

[25] G. Hu, Y. Liu, J. Zhao, S. Cui, Z. Yang, Y. Zhang, Bioelectrochemistry 69 (2006) 254.

[26] D. Narasaiah, C.K. Mitra, Anal. Lett. 25 (1992) 443.

[27] D. Narasaiah, C.K. Mitra, Electroanalysis 5 (1993) 589.

[28] S.K. Cha, B.K. Ahn, J.U. Hwang, H.D. Abruna, Anal. Chem. 65 (1993) 1564.

[29] A. Richard, A. Margaritis, Crit. Rev. Biotechnol. 21 (2001) 219. 
[30] Z. Cheng, S.H. Teoh, Biomaterials 25 (2004) 1991.

[31] A.-M. Chiorcea Paquim, V.C. Diculescu, T.S. Oretskaya, A.M. Oliveira Brett, Biosens. Bioelectron. 20 (2004) 933.

[32] H. Chen, C.K. Heng, P.D. Puiu, X.D. Zhou, A.C. Lee, T.M. Lim, S.N. Tan, Anal. Chim. Acta 554 (2005) 52.

[33] S. Campuzano, M. Pedrero, C. Montemayor, E. Fatás, J.M. Pingarrón, J. Electroanal. Chem. 586 (2006) 112.
[34] Y. Hou, S. Helali, A. Zhang, N. Jaffrezic-Renault, C. Martelet, J. Minic, T. Gorojankina, M.-A. Persuy, E. Pajot-Augy, R. Salesse, F. Bessueille, J. Samitier, A. Errachid, V. Akimov, L. Reggiani, C. Pennetta, E. Alfinito, Biosens. Bioelectron. 21 (7) (2006) 1393.

[35] Y. Li, X. Lin, Sens. Actuators B 115 (2006) 134.

[36] Y. Sha, L. Qian, Y. Ma, H. Bai, X. Yang, Talanta 70 (2006) 556.

[37] A.M. Oliveira Brett, A.-M. Chiorcea, Langmuir 19 (2003) 3830. 